\title{
Glucocorticoid Administration Improves Aberrant Fear-Processing Networks in Spider Phobia
}

\author{
Masahito Nakataki ',2,9, Leila M Soravia',9, Simon Schwab', Helge Horn', Thomas Dierks', Werner Strik', \\ Roland Wiest $^{3,4}$, Markus Heinrichs ${ }^{5,6}$, Dominique J-F de Quervain ${ }^{7}$, Andrea Federspiel ${ }^{1,10}$ and \\ Yosuke Morishima*, I,8,10 \\ 'Translational Research Center, University Hospital of Psychiatry, University of Bern, Bern, Switzerland; '2Department of Psychiatry, University of \\ Tokushima, Tokushima, Japan; ${ }^{3}$ Departments of Neurology and Clinical Research, Inselspital, University Hospital of Bern, Bern, Switzerland; \\ ${ }^{4}$ Department of Diagnostic and Interventional Neuroradiology, Inselspital, University Hospital of Bern, Bern, Switzerland; ${ }^{5}$ Department of \\ Psychology, Laboratory for Biological and Personality Psychology, University of Freiburg, Freiburg, Germany; ${ }^{6}$ Freiburg Brain Imaging Center, \\ University Medical Center, University of Freiburg, Freiburg, Germany; ${ }^{7}$ Division of Cognitive Neuroscience University of Basel, Basel, Switzerland; \\ \&apan Science and Technology Agency, PRESTO, Saitama, Japan
}

\begin{abstract}
Glucocorticoids reduce phobic fear in patients with anxiety disorders. Previous studies have shown that fear-related activation of the amygdala can be mediated through the visual cortical pathway, which includes the fusiform gyrus, or through other pathways. However, it is not clear which of the pathways that activate the amygdala is responsible for the pathophysiology of a specific phobia and how glucocorticoid treatment alleviates fear processing in these neural networks. We recorded the brain activity with functional magnetic resonance imaging in patients with spider phobia, who received either $20 \mathrm{mg}$ of cortisol or a placebo while viewing pictures of spiders. We also tested healthy participants who did not receive any medication during the same task. We performed dynamic causal modelling (DCM), a connectivity analysis, to examine the effects of cortisol on the networks involved in processing fear and to examine if there was an association between these networks and the symptoms of the phobia. Cortisol administration suppressed the phobic stimuli-related amygdala activity to levels comparable to the healthy participants and reduced subjective phobic fear. The DCM analysis revealed that cortisol administration suppressed the aberrant inputs into the amygdala that did not originate from the visual cortical pathway, but rather from a fast subcortical pathway mediated by the pulvinar nucleus, and suppressed the interactions between the amygdala and fusiform gyrus. This network changes were distinguishable from healthy participants and considered the residual changes under cortisol administration. We also found that the strengths of the aberrant inputs into the amygdala were positively correlated with the severity of spider phobia. This study demonstrates that patients with spider phobia show an aberrant functional connectivity of the amygdala when they are exposed to phobia-related stimuli and that cortisol administration can alleviate this fear-specific neural connectivity.

Neuropsychopharmacology (2017) 42, 485-494; doi:I0.1038/npp.20 I6.207; published online I2 October 2016
\end{abstract}

\section{INTRODUCTION}

The amygdala processes emotional information, and the processing of emotional information enables organisms to interrupt ongoing actions and redirect attentional resources toward unforeseen threats (Dolan and Vuilleumier, 2003; Pessoa and Adolphs, 2010; Phelps, 2006). Specifically, the automatic processing of fearful stimuli plays a crucial role in the protection against threats (Soares and Ohman, 1993; Mogg et al, 1997; Phelps and LeDoux, 2005). However, neutral stimuli could be erroneously associated with fear, as

\footnotetext{
*Correspondence: Dr Y Morishima, Translational Research Center, University Hospital of Psychiatry, University of Bern, Bolligenstrasse II I, Bern 3000, Switzerland, Tel: +4I 3193287 05, Fax: +41 319309961 , E-mail: yosuke.morishima@puk.unibe.ch

${ }^{9}$ Shared first authorship.

${ }^{10}$ Shared senior authorship.

Received 2 June 2016; revised I September 2016; accepted 14 September 2016; accepted article preview online 20 September 2016
}

in the case of a specific phobia, and the maladaptive association provokes phobia-related responses to the neutral stimuli (Hartley and Phelps, 2012; de Quervain and Margraf, 2008; Soares and Ohman, 1993). A specific phobia is the most common anxiety disorder and is characterized by persistent and excessive fear that is cued by the anticipation or presence of a specific object or situation in DSM-IV (American Psychiatric Association, 1997). The exposure to, or even the anticipation of, the phobic stimulus invariably provokes the retrieval of stimulus-associated fear memories, leading to an immediate fear response (Hartley and Phelps, 2012; de Quervain and Margraf, 2008). Because the amygdala is involved in the response to fearful stimuli, and to the acquisition and maintenance of aversive memories (Becker et al, 2012; Feinstein, 2013; Pape and Pare, 2010), it is considered to play a crucial role in the pathophysiology of specific phobias (Etkin and Wager, 2007; Hariri et al, 2005). Aberrant amygdala hyperactivation is frequently reported in anxiety disorders, including specific phobias (Veltman et al, 
2004; Ahs et al, 2009; Lipka et al, 2013), and those can be improved by pharmacological intervention and cognitive behavioral therapy (Lipka et al, 2013; Schienle et al, 2009; Labuschagne et al, 2010; Hauner et al, 2012; Eckstein et al, 2014). Therefore, the dysfunction of the amygdala is believed to be the central pathophysiological mechanism of specific phobias.

Glucocorticoid hormones (cortisol in humans) are released from the adrenal cortex. Their release is enhanced in response to a stressor to enable responses to any environmental hazards and glucocorticoid receptors are ubiquitously expressed in the brain (Myers et al, 2014). In animals and healthy humans, glucocorticoids reduce memory retrieval (de Quervain et al, 1998, 2000) and recent clinical studies have found that in patients with chronic post-traumatic stress disorder, social phobia, spider phobia, and acrophobia, the administration of glucocorticoids reduces the ability to recall traumatic or fearful memories, and enhances the extinction of such memories (Aerni et al, 2004; van Peer et al, 2010; Soravia et al, 2006; de Quervain et al, 2011). A recent neuroimaging study showed that glucocorticoid administration altered amygdala reactivity related to the retrieval of aversive memories, the presentation of emotionally salient face pictures and predicted reward (BuadesRotger et al, 2016; van Marle et al, 2013; Montoya et al, 2014). Because functional MRI (fMRI) signals reflect afferent inputs from other brain regions (Goense and Logothetis, 2008), the glucocorticoid-mediated suppression of amygdala activity likely reflects the suppression of neural inputs into the amygdala during the processing of fearful stimuli. Fearinduced amygdala activation can originate from several brain regions, including the fusiform gyrus, orbitofrontal cortex, anterior cingulate cortex, and subcortical structures, such as the pulvinar nucleus through distinct pathways, and, in particular, the pathways from the fusiform gyrus and the pulvinar nucleus could serve as the bottom-up pathways of information processing (Morris et al, 1999; Pessoa and Adolphs, 2010; Van Le et al, 2013). However, the neural pathway involved in the pathological processing of phobiarelated information remains unknown. In the current study, we investigated how glucocorticoids affect neural circuits for fear processing in patients with spider phobia. More specifically, we focused on the pathways through the visual cortical pathway, including the lingual and fusiform gyrus, and the pulvinar nucleus to the amygdala. We utilized fMRI to record the neural activity in response to pictures of spiders in patients with spider phobia, who received either cortisol or placebo, and in control subjects who did not receive any medication. As shown in previous studies (Aerni et al, 2004; Soravia et al, 2014), cortisol treatment improves phobic symptoms, but patients still exhibits a significant level of phobic response. To investigate neural correlates of residual symptoms, we compared the phobic patient groups with the healthy controls. We then used dynamic causal modelling (DCM) to investigate the functional interaction between the neural circuits that process phobia-related stimuli and to determine the source(s) of activation for a particular brain region (Friston et al, 2003). Using the model comparison approach, we aimed to elucidate the functional interactions in the regions of the amygdala that become hyperactive in specific phobias and to identify the neural target of glucocorticoid treatment.

\section{MATERIALS AND METHODS}

\section{Subjects}

This study consisted of three subject groups: patients with spider phobia who received either $20 \mathrm{mg}$ of cortisol or placebo in a double-blind and randomized way, and healthy participants who did not receive any medication. The patients were selected from a cohort of our previous clinical trial, investigating the effectiveness of cortisol treatment and cognitive behavioral therapy on spider phobia treatment (Soravia et al, 2014; clinicaltrials.gov NCT01574014). The subjects were recruited via advertisement. The patients were diagnosed based on the Diagnostic and Statistical Manual of Mental Disorders, fourth edition (American Psychiatric Association, 1997), using a computer-based structured clinical interview (DIA-X; Essau et al, 1999) and the Composite International Diagnostic Interview (RubioStipec et al, 1991). The German version of the Fear of Spider Questionnaire (FSQ; Szymanski and O'Donohue, 1995) was further used to assess the fear of spiders. This assessment was used to confirm that the spider phobia symptoms that were measured in the patients were significantly more severe than those in the control subjects $(t(48)=12.34, p<0.001)$. Healthy participants were screened by the SCL-90-R (The Symptom Checklist-90-Revised) questionnaire (Franke, 1995) and the FSQ to confirm that the participants were not affected by mental illness, including spider phobia (three healthy controls had no FSQ data). We also confirmed no previous history of psychiatric disorders. The exclusion criteria included a history of head trauma, acute or chronic medical conditions, a recent history of systemic or oral glucocorticoid therapy, psychiatric disorders other than a specific phobia for spiders, psychotropic drug treatment, smoking of $>15$ cigarettes per day, neurological diseases, current drug or alcohol abuse, any contraindication to MRI, pregnancy, and the use of hormonal contraceptives. After a consultation that involved a complete description of the study, written informed consent was obtained from each subject. The study was approved by the ethics committee of the Canton of Bern, Switzerland (Nr. 161/07), and the Swiss agency for the authorization and supervision of therapeutic products (Swissmedic) in accordance with the Declaration of Helsinki. After the diagnostic phase, the included patients were randomly assigned to treatment groups in a doubleblind and placebo-controlled manner. Eighteen patients were assigned for each patient group (11 female for cortisol group; and 15 female for placebo group) and 27 healthy control subjects were included after the assessment of inclusion criteria. Two patients and three healthy control subjects were excluded due to missing data, and one patient was excluded because of an ineffective elevation in salivary cortisol levels in response to the cortisol administration. We performed general linear model analysis with remaining 57 subjects to increase the sensitivity and reliability of region of interests (ROI) specification (cortisol group: $N=17$ (10 females); and placebo group: $N=17$ (14 females)) and 24 healthy control subjects (13 females). We used only the female participants for the remaining analysis, including the analysis of behavioral, pharmacological, and DCM data to avoid any gender differences in the responsiveness to fearful stimuli (demographic data are shown in Table 1). After the GLM

Neuropsychopharmacology 
Table I Demographics and Descriptive Statistics of Behavioral Data

\begin{tabular}{|c|c|c|c|}
\hline Group & Cortisol & Placebo & $\begin{array}{l}\text { Healthy } \\
\text { control }\end{array}$ \\
\hline Number of female subjects & 10 & 14 & 13 \\
\hline Age (years) & $31.3 \pm 11.2$ & $26.6 \pm 11.2$ & $27.2 \pm 6.8$ \\
\hline $\mathrm{BMI}$ & $22.7 \pm 4.9$ & $22.8 \pm 3.7$ & $22.1 \pm 2.4$ \\
\hline FSQ & $75.3 \pm 24.4^{\mathrm{a}}$ & $77.9 \pm 11.7^{\mathrm{a}}$ & $11.6 \pm 14.0$ \\
\hline \multicolumn{4}{|l|}{ Pre-scan } \\
\hline STAl state & $41.2 \pm 13.9$ & $35.6 \pm 8.3$ & $31.7 \pm 3.9$ \\
\hline \multicolumn{4}{|l|}{ Mid-scan, picture task } \\
\hline VAS fear: phobic pictures & $2.9 \pm 0.6^{\mathrm{a}, \mathrm{b}}$ & $3.3 \pm 0.5^{\mathrm{a}}$ & $1.7 \pm 0.8$ \\
\hline VAS fear: negative pictures & $1.8 \pm 0.7$ & $1.7 \pm 0.5$ & $1.9 \pm 0.4$ \\
\hline VAS fear: neutral pictures & $1.1 \pm 0.1$ & $1.0 \pm 0.1$ & $1.0 \pm 0.1$ \\
\hline VAS fear: animal pictures & $1.2 \pm 0.2$ & $1.1 \pm 0.1$ & $1.2 \pm 0.2$ \\
\hline \multicolumn{4}{|l|}{ Post scan } \\
\hline STAl state & $33.8 \pm 10.7$ & $36.9 \pm 14.1$ & $29.0 \pm 5.5$ \\
\hline VAS fear retrospect ${ }^{c}$ & $64.3 \pm 19.1^{d, e}$ & $78.4 \pm 11.2^{d}$ & $26.5 \pm 25.5$ \\
\hline \multicolumn{4}{|l|}{$\begin{array}{l}\text { Cortisol concentration of saliva } \\
\text { (nmol/l) }\end{array}$} \\
\hline Before administration & $9.9 \pm 5.4$ & $9.9 \pm 5.6$ & \\
\hline $\begin{array}{l}60 \text { min after } \\
\text { administration }\end{array}$ & $40.3 \pm 22.0^{b}$ & $8.0 \pm 4.5$ & \\
\hline $\begin{array}{l}120 \text { min after } \\
\text { administration }\end{array}$ & $58.2 \pm 43.3^{b}$ & $5.1 \pm 2.6$ & \\
\hline
\end{tabular}

Abbreviations: BMI, body mass index; FSQ, Fear of Spider Questionnaire (German version); mid-scan: each picture had to be rated in the scanner on a scale from I (no fear) to 4 (maximum fear); STAl, State-Trait Anxiety Inventory; VAS, visual analog scale.

All data are presented as the means $\pm \mathrm{SD}$.

a $p<0.00$ I compared with control group.

${ }^{b} p<0.00$ I compared with placebo group.

'After the scanning session, subjects were asked to rate the experienced emotion during the viewing of spider pictures in the scanner retrospectively on a scale from 0 to 100 percent.

${ }_{p} p<0.05$ compared with control group.

e $p<0.05$ compared with placebo group.

analysis, we excluded one patient from the cortisol group and two healthy control subjects due to fMRI signal dropout in the individual ROI.

All patients were offered the opportunity to attend an exposure-based short-term group therapy session after the conclusion of the experiment. The three groups did not significantly differ in age or BMI (Table 1 ). The two patient groups did not differ in any of the clinical characteristics or baseline measurements assessed on the day of the experiments (Table 1).

\section{Procedure and Measurements}

The experiments were conducted at the Institute of Diagnostic and Interventional Neuroradiology, University Hospital of Bern between 1400 and 1700 hours. The female subjects were tested within the luteal phase of their menstrual cycle. Only females with a natural menstrual cycle were included in the study and tested during their luteal phase in order to be comparable to male subjects, regarding the cortisol stress response (Kirschbaum et al, 1999). The patients and healthy control subjects underwent the same experimental procedure except for the diagnostic interview, substance administration, and collection of saliva samples. The healthy control subjects answered the SCL-90-R questionnaire instead. Saliva samples were collected to confirm the effectiveness of the cortisol administration in the patient group. On arrival, all the participants were informed about the procedure and were asked to complete the State-Trait Anxiety Inventory (Spielberger et al, 1970) and rate their actual subjective anxiety, physical discomfort, and avoidance behavior on a visual analog scale (VAS) ranging from 0 (no symptoms) to 100 (maximal symptoms). In addition, the first saliva sample was collected using a Salivette (Sarstedt, Rommelsdorf, Germany). After the oral administration of $20 \mathrm{mg}$ of cortisol (Galepharm, Küsnacht, Switzerland) or placebo, the patients rested for $\sim 30 \mathrm{~min}$. Additional saliva samples were collected 60 and 120 min after drug administration to confirm an elevation in their cortisol levels. The details of the picture task and fMRI scanning protocols are described in the next section. After the MRI session, all subjects completed a VAS questionnaire. In addition, the participants retrospectively rated their fear levels on a visual scale from 0 to 100 while observing the spider pictures in the scanner. We also inquired about any side effects that they may have experienced because of the glucocorticoid or placebo administration. The saliva samples were stored at $-20^{\circ} \mathrm{C}$ until used for biochemical analysis. The free salivary cortisol concentrations were analyzed using a commercially available chemiluminescence immuno-assay (cortisol: CLIA; IBL-Hamburg, Germany).

\section{MRI Data Acquisition and fMRI Data Analysis}

A brief summary of the MRI data acquisition, the cognitive task and fMRI data analysis is included here, and the details can be found in the Supplementary Methods. Structural T1-weighted images and oxygenation level-dependent T2-weighted gradient echo planar imaging images were acquired using a $3 \mathrm{~T}$ Siemens Magnetom Trio scanner (Erlangen, Germany), equipped with a 12-channel radio frequency head coil, although the participants went on the picture task (Figure 1a). The fMRI data analyses were performed using SPM8 and DCM12 (http://www.fil.ion.ucl. ac.uk/spm). After the preprocessing of the fMRI data, the first-level single-subject analysis was conducted with a general linear model (GLM) framework, and the GLM included six regressors: phobic, negative, animal, neutral, scramble 1 , and scramble 2 . The second-level random effects analysis was performed as a $3 \times 6$ flexible factorial design with group (placebo, cortisol, and healthy subjects) as a between-subject factor and task condition (phobic, negative, animal, neutral, scramble 1 , and scramble 2) as a withinsubject factor (Supplementary Table S1). To exclude the confounding effect of a gender difference in the responsiveness to the fearful stimuli, we have also included a regressor encoding gender for each group. The three ROIs for the DCM analysis (ie, right amygdala, right fusiform gyrus, and 
a

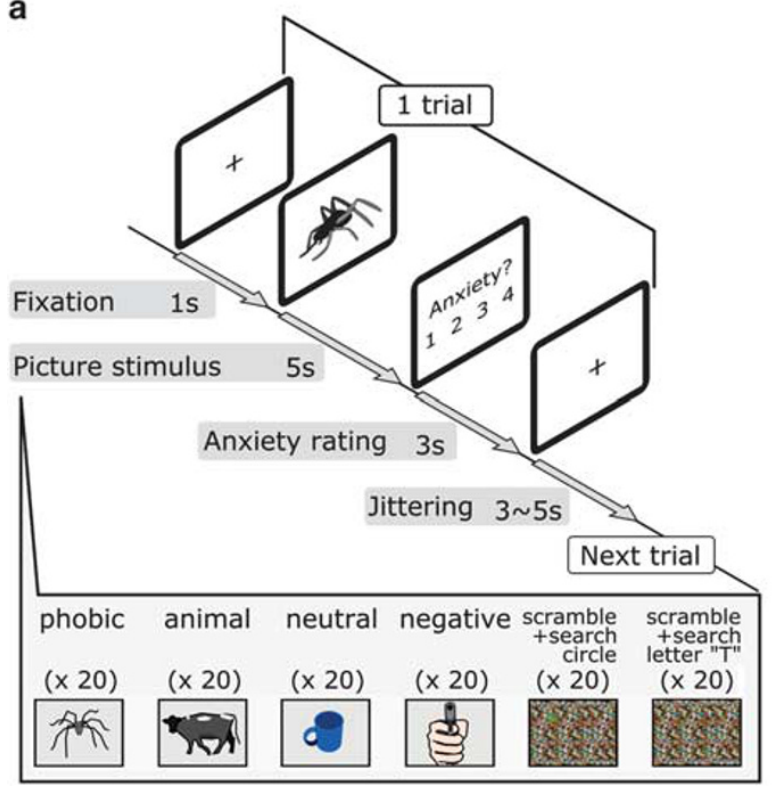

b
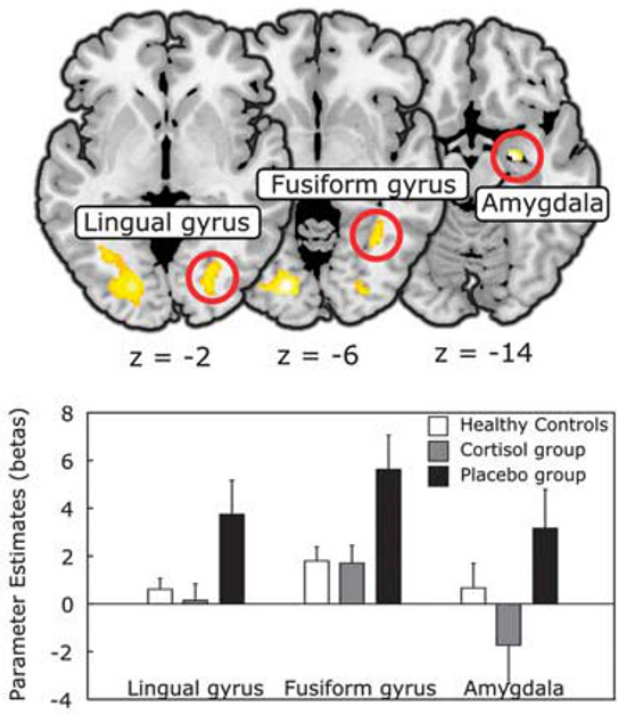

Figure I The cognitive task and regional activation during spider picture presentation. (a) The timeline of the picture task is shown. Each trial includes the picture presentation and rating periods. (b) Top: the brain areas that showed higher levels of activation during the spider picture presentation in the placebo group compared with the cortisol group are shown, as derived from a 3 (group) $\times 6$ (condition) factorial ANOVA. For these representative images, all the voxels survived at $p<0.00$ I uncorrected are depicted. Bottom: a plot of the parameter estimates for phobia-related picture presentation in the right lingual gyrus, right fusiform gyrus, and right amygdala is shown. The error bars indicate SE across subjects. The bar plots are representative images and were not the basis of any inferences in this study.

a

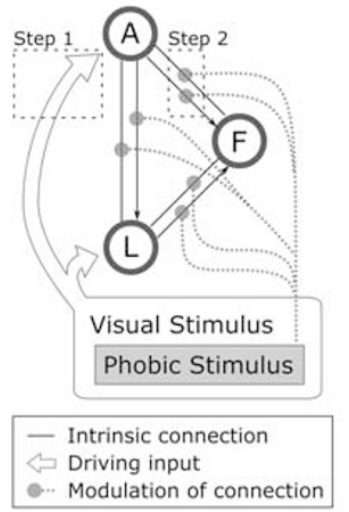

b

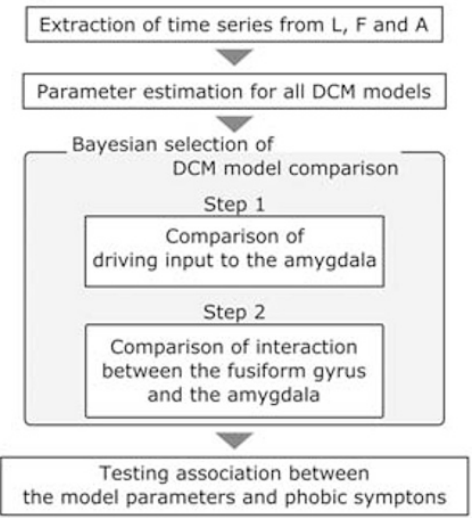

Figure 2 DCM model structure and the two steps approach of DCM model comparison. (a) The structure of the networks used for DCM analysis are shown. L, F, and A were supposed to have intrinsic connections to each other, although the driving inputs and modulation of the connections by the phobia-related stimulus (spider picture) differed between models. To test the effect of cortisol on this neural network, Bayesian model selection was applied for a family-level model comparison. Two different features were determined separately and were illustrated as dashed squares (step I and step 2). (b) An overview of the DCM analysis and Bayesian model selection is shown. At step I, all the models were compared according to the pattern of the driving input into the amygdala. At step 2, the models for the winning family in step I were compared according to the pattern of interaction between the fusiform gyrus and the amygdala.

right lingual gyrus) were functionally defined as the areas exhibiting a higher activation in the second-level results (Figure 1b). We chose the right hemisphere ROIs because the cortisol-dependent suppression of fear-related activation was

more prominent on the right side of the fusiform gyrus and amygdala than on the left side in the current data set (Supplementary Table S2). We extracted a BOLD time series from the ROIs and entered them into the estimation of specified DCM models (Figure 2a; Supplementary Figure S1). We created 48 DCM models for each subjects, comprised of the possible combinations of directed connectivity among the ROIs and driving inputs to the ROIs. We then compared the models to identify the best-fit structure of the fearprocessing network (Stephan et al, 2010). We employed a two-step approach for the model comparison (Figure $2 \mathrm{a}$ and b). First, we examined whether the inputs into the amygdala were necessary to generate a fear-related amygdala response (Supplementary Figure S2). Then, we looked for an interaction between the amygdala and the fusiform gyrus (Supplementary Figure S3). Finally, we performed Bayesian model averaging to obtain the parameter estimates from the fear network model (Stephan et al, 2010). We have performed the DCM model analysis of the pulvinaramygdala pathway as for the visual cortical pathway (Supplementary Figure S4). Details can be found in the Supplementary Methods.

\section{Statistics}

Group differences in demographics, clinical characteristics, and baseline level of salivary cortisol (before substance administration) were analyzed with an ANOVA and unpaired $t$-tests. The effects of the cortisol administration on salivary cortisol concentrations, VASs and the extracted beta values were analyzed with two-way repeated measures ANOVAs, using 'treatment' or 'group' as the between-subject 


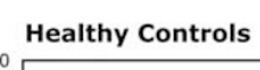

Cortisol
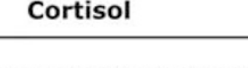

Placebo
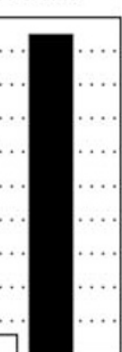

L LA b
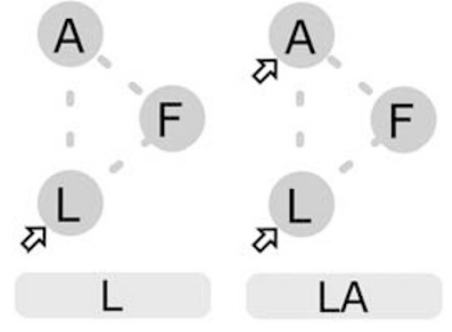

Figure 3 The first step of the model comparison of driving inputs. (a) The exceedance probabilities of the $L$ and $L A$ model families are shown. Family $L$ has visual inputs only into the lingual gyrus, whereas family LA has inputs into both the lingual gyrus and the amygdala. The group of patients treated with placebo (right) showed a considerably higher exceedance probability of the LA model family than that of the L model family. By contrast, the cortisol group (center) and the healthy control group (left) showed considerably higher exceedance probability of the L model family than that of the LA model family. (b) The diagrams of the family L and family LA show the structures of the driving inputs (empty arrow). The modulation of the connection and intrinsic connection are not shown for comprehensibility. Three regions included in the DCM models are denoted by L (lingual gyrus), F (fusiform gyrus), and A (amygdala).

factor and 'time points' as the within-subject factor. As the salivary cortisol data did not follow a normal distribution, we used the Wilcoxson rank-sum test to compare the difference between the groups at a specific time point. A GreenhouseGeisser correction was used to correct violations of sphericity. Subsequent unpaired $t$-tests were used to analyze the treatment effects at specific time points. To analyze the behavioral responses in the MRI scanner, we used a mixed model analysis with 'Ime4' and 'ImerTest' packages implemented in R (https://www.r-project.org). We used a full factorial design, including each subject group and each picture type, and their interaction effects as fixed effects and an individual subject factor as random effects. All the tests were two tailed, and a probability $<0.05$ was considered to be statistically significant.

\section{RESULTS}

During the presentation of the pictures of spiders, phobic patients who received cortisol rated their subjective fear as being significantly lower than that of the placebo group $(p<0.001)$, but still significantly higher than that of the control group ( $p<0.001$; Table 1, Supplementary Table S1). After the scanning session, subjects were asked to rate their perceived fear in response to the spider pictures, and cortisol administration significantly reduced the subjective fear ratings $(t(22)=-2.6, p=0.016$; Table 1$)$. There was no significant effect of cortisol on the fear ratings for negative, neutral, and animal pictures or on the state of anxiety unrelated to spider phobia before and after the scanning session ( $p>0.1$; Table 1). Consistent with the behavioral results, the activation of the amygdala in response to the spider pictures in the placebo-treated patients was significantly higher than that of the cortisol-treated patients (Figure 1b; Supplementary Table S2) and significantly higher compared with all the other stimuli (Supplementary Table S2). In addition, we found a significantly increased activation level in the bilateral lingual gyrus and right fusiform gyrus during the phobic spider picture presentation in the placebo group compared with the cortisol group (Figure 1b; Supplementary Table S2). We confirmed that the salivary cortisol levels were increased in the patients receiving $20 \mathrm{mg}$ of cortisol by a significant time $x$ group interaction effect $(\mathrm{F}(1.4,38.8)=19.78 ; p<0.001)$. As expected, the post hoc comparisons revealed an increased salivary cortisol level at both 60- and 120-min post-cortisol administration in the cortisol group compared with the placebo group $(p<0.001$; Table 1$)$. We also observed the decay of cortisol levels over time in the placebo group, and this decay can be explained by the immediate elevation of cortisol due to exposure to laboratory environments and changes reflecting circadian rhythm (Chan and Debono, 2010; Dickerson and Kemeny, 2004).

\section{Dissecting the Source of the Amygdala Activation}

The DCM analysis was performed to determine whether the visual cortical pathway of the lingual and fusiform gyri mediates the observed amygdala activation in response to spider pictures in spider-phobic patients. Therefore, we categorized the DCM models into two families: family L has visual inputs only into the lingual gyrus and family LA has visual inputs into both the lingual gyrus and the amygdala (Figure 3b; Supplementary Figure S2). We compared the DCM model families and found that the placebo-treated patients showed a considerably higher exceedance probability in the LA model family than in the L model family (Figure 3a). In contrast, the cortisol-treated patients and healthy control groups showed a considerably higher exceedance probability of the L model family than in the LA model family. The DCM model comparison of the placebo group data suggests that activation in the amygdala could be mediated not only by the visual cortical areas, but also by other brain regions. Inputs of visual information to the amygdala are known to be mediated by two pathways: a faster subcortical pathway (pulvinar-amygdala) and a slower cortical pathway (thalamus-visual cortex-amygdala). These two visual processing pathways are well characterized in animals (Phelps and LeDoux, 2005; Van Le et al, 2013) and are supported by human studies (Luo et al, 2007). Thus, we have additionally tested whether the amygdala activation in the placebo group can be mediated by the pulvinar nucleus. We have compared two DCM models. First, the amygdala activation was mediated by the activation of the pulvinar nucleus. Second, the amygdala activation is independent of 

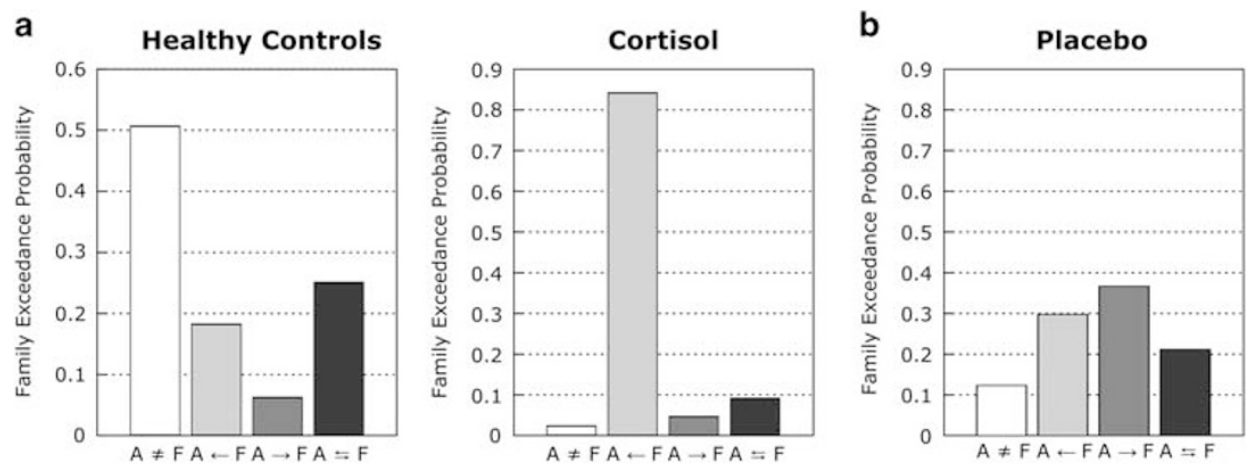

c
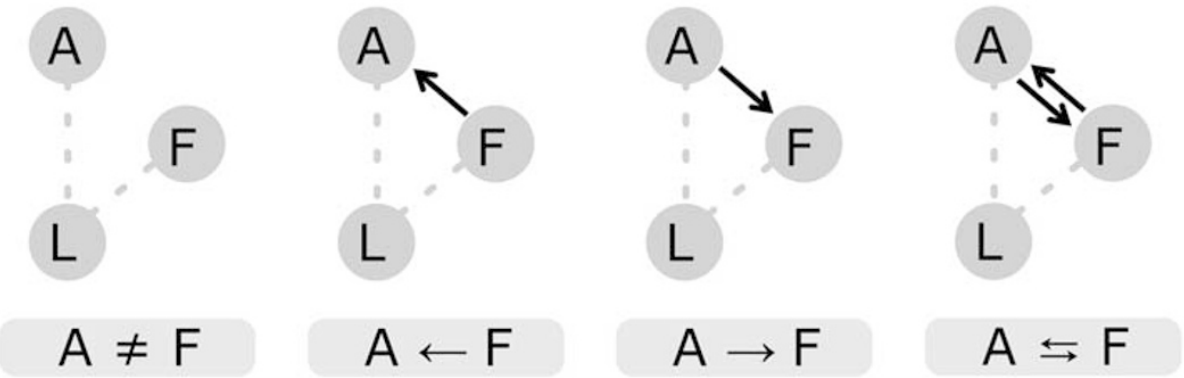

Figure 4 The second step model comparison of the modulatory interaction between the amygdala and the fusiform gyrus. (a) This is shown according to the pattern of interactions between the fusiform gyrus ( $F$ ) and the amygdala (A). The healthy controls have no modulatory effect on the connection between the amygdala and the fusiform gyrus. The cortisol group had a modulatory connection from the fusiform gyrus to the amygdala. (b) In the placebo group, no prominent family of modulatory connection was observed. (c) The diagram of family $A \neq F, A \leftarrow F, A \rightarrow F$, and $A \rightleftarrows F$ is shown. Family $A \neq F$ does not have $a$ modulation of connection on a connection between $F$ and $A$. Family $A \leftarrow F$ and family $A \rightarrow F$ have a modulation of connection on a unilateral connection from $F$ to $A$ and from $A$ to $F$, respectively. Family $A \rightleftarrows F$ has a modulation of a connection on the bilateral connections between $F$ and $A$. The figure shows the structures of the modulation of connection (solid arrow). The other modulations of connection and intrinsic connection are not shown for comprehensibility.

pulvinar activation. We then compared these two models and found that the amygdala activation is mediated by the pulvinar nucleus in the placebo-treated patients (Supplementary Figure S4). These results suggest that the amygdala in phobic patients has, in addition to inputs from the visual cortical pathway with involvement of the lingual and fusiform gyrus, a driving visual input mediated by the pulvinar nucleus, and that cortisol normalizes these additional amygdala inputs.

\section{Residual Interactions Between Amygdala and Fusiform Gyrus After Cortisol Treatment}

We next aimed to examine whether there is any residual change in the modulation of the connection during phobic picture presentation in cortisol-treated patients. As we have shown, cortisol treatment suppressed the fear rating of spider pictures, but the score was still higher than that of healthy individuals, implying that there are residual neural changes in the patients of the cortisol group. Because both the cortisol and the healthy control groups have driving inputs only into the lingual gyrus, we compared 24 models belonging to the L model family. Because previous studies have shown that the perception of phobic visual stimuli involves both the amygdala and the fusiform gyrus (Ahs et al, 2009; Lipka et al, 2013; Straube et al, 2006), we focused on the pattern of interactions between the fusiform gyrus (F) and amygdala (A) and classified each model to one of the four modulatory pattern families (Figure 4c; Supplementary Figure S3). We found that the model family with the modulation of connectivity from the fusiform gyrus to the amygdala (A $\leftarrow \mathrm{F}$ ) was the best-fit model family for the cortisol group, whereas the no interaction model family $(\mathrm{A} \neq \mathrm{F})$ was the bestfit model family for the healthy control group (Figure 4a). For the placebo group, there was no clear difference in the exceedance probability among the four model families (Figure 4b).

Finally, we calculated the parameters of the modulation of connection for the DCM models (Figure 5; and Supplementary Table S3 for the numerical representation). We found two significant changes: (1) a significant difference in the modulation of the connection between the fusiform gyrus and the amygdala between the cortisol and placebo groups (one-way ANOVA, post hoc test $p<0.01$ ); (2) a significant negative modulation of the connection between the amygdala and the lingual gyrus in the placebo-treated patients relative to the cortisol-treated patients and healthy controls (one-way ANOVA, post hoc test $p<0.05$ ). Here, the positive values indicate that the increased activation of a source region enhances the activation of a target region during the presentation of a phobic stimulus, whereas the negative values indicate that the increased activation of the source suppresses the target. The results suggest that cortisol administration turns fear-related positive modulation from the fusiform gyrus to the amygdala to negative modulation. 
Healthy control group

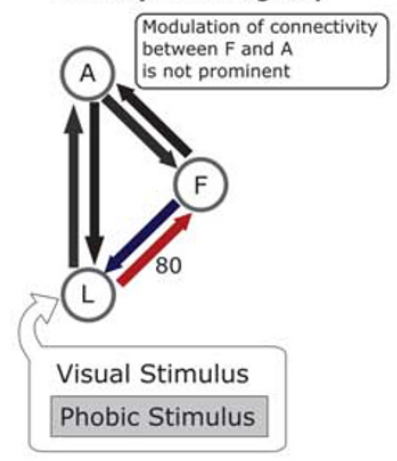

Cortisol group

Activity of A against phobic stimulus $\Rightarrow$

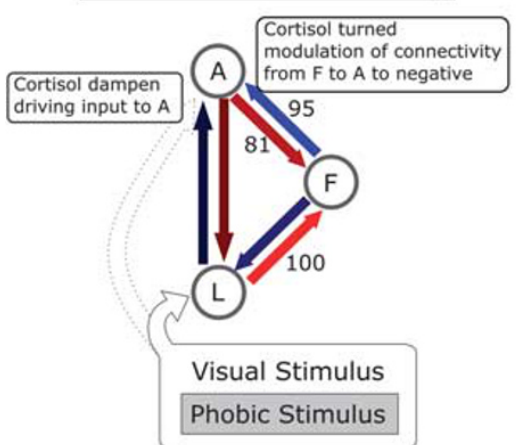

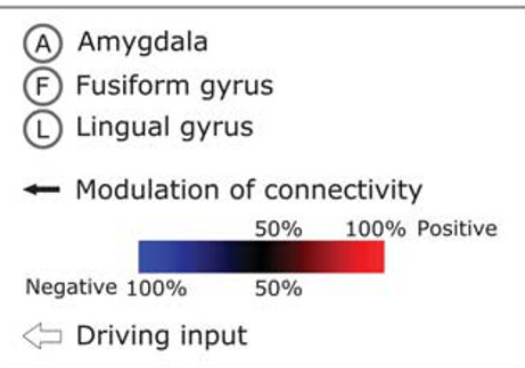

Placebo group
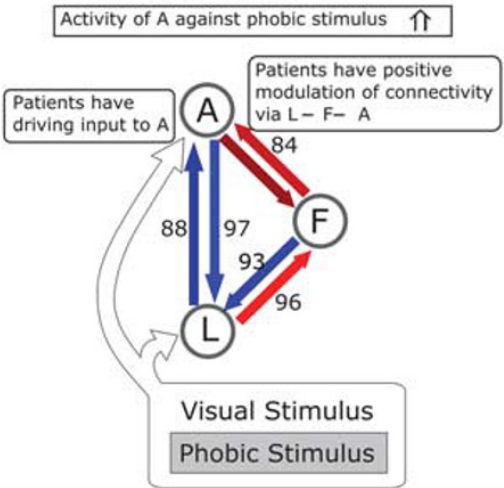

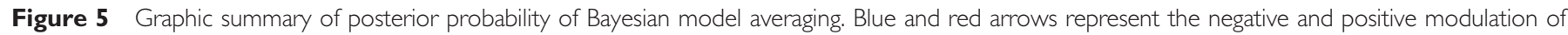

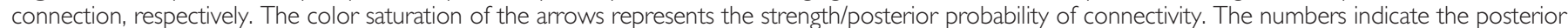
probability of each modulatory connectivity (only posterior probability $>80 \%$ is shown).

\section{The Association of Amygdala Inputs with Phobia- Related Symptoms}

We further investigated the relevance of the DCM model parameters to phobia-related symptoms in the patient groups. Because we have found that cortisol treatment suppresses both the driving input into the amygdala and the fear rating of spider picture presentations, we examined the association between the driving inputs into amygdala and the FSQ. We found a significant positive correlation between the driving inputs into amygdala during spider picture presentation and the FSQ score in the placebo group $(r=0.63, p=0.017)$, although the correlation was not statistically significant in the cortisol group (Figure 6). In contrast, we did not find a correlation between the FSQ score and the regional BOLD activity estimated in the GLM analysis $(r=0.19, p=0.49)$. The results suggest that the severity of phobia-related symptom is associated with the strength of the driving inputs into amygdala, but not with the regional activation, in other words, the sum of the total inputs into the amygdala.

\section{DISCUSSION}

In this study, we demonstrated that cortisol treatment suppresses the neural inputs into the amygdala during the processing of phobia-related information and that driving inputs into the amygdala are positively correlated with the severity of spider phobia symptoms. We constructed a set of neural circuit models consisting of the amygdala, fusiform gyrus, and lingual gyrus, and compared the models according to the pattern of interactions among the different brain regions. The model comparison revealed a mixture of pathological neural signatures of the phobia and of the pharmacological effects of glucocorticoids. The patients treated with the placebo differed from the other groups, in that they had additional driving inputs into the amygdala for phobia-related stimuli, mediated by the pulvinar nucleus. Acute cortisol administration in the patients with spider phobia reduced these driving inputs into the amygdala while they were viewing phobic pictures. However, cortisol-treated patients still exhibited abnormal residual modulation of the connection between the fusiform gyrus and the amygdala.

Previous studies have found an abnormal amygdala hyperactivation in patients with phobias (Veltman et al, 2004; Ahs et al, 2009; Lipka et al, 2013), which was downregulated after treatment (Lipka et al, 2013; Schienle et al, 2009; Hauner et al, 2012). Our current results have advanced the understanding of the pathophysiology of specific phobia. We have shown that the severity of a spider phobia is specifically associated with the driving inputs into amygdala, but not with the regional BOLD activation of the amygdala. To narrow down the source of the driving inputs in the current model, we have exploited an approach established by Hurlemann's study to localize the amygdala ROI in the subnucleus of the amygdala (Hurlemann et al, 2008 ), and $71.0 \%$ of the voxels of the amygdala ROI were localized in the basolateral part of the amygdala, which 

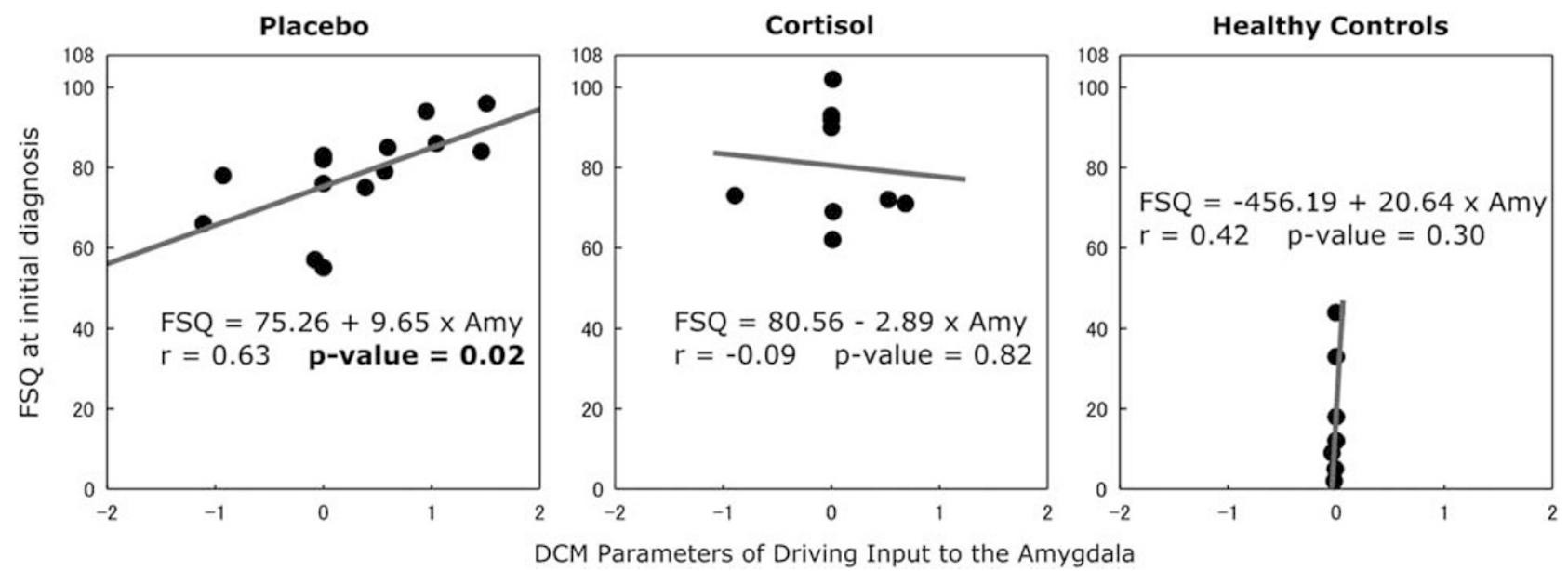

Figure 6 The association between the DCM parameter of the driving input into the amygdala and the severity of phobic symptom. There is a significant positive correlation between the FSQ score at the initial diagnosis and the DCM parameters of the driving input into the amygdala in the placebo group $(p=0.017)$, although no significant correlations in the cortisol and healthy groups were found. (For healthy controls three subjects had no FSQ data.)

putatively receives inputs from cortical and subcortical regions. Importantly, it has been shown that the basolateral part of the amygdala interacts with the hippocampus in mediating glucocorticoid effects on the retrieval of emotionally arousing information (de Quervain et al, 2009). In the current study, we have demonstrated that a driving input to the amygdala is mediated by the pulvinar nucleus. However, others have demonstrated that the rostral anterior cingulate cortex suppresses the amygdala activity during the processing of emotionally conflicting information (Etkin et al, 2006), and that amygdala-hippocampus interactions are crucial during the processing of emotionally salient stimuli (Fastenrath et al, 2014) and in mediating glucocorticoid effects on the retrieval of emotionally arousing information (de Quervain et al, 2009). Those studies suggest the involvement of other brain regions in processing fear information in the amygdala. Although models involving such higher cognitive areas are beyond the current study, research on large-scale neural networks will be important for the identification of the networks that are responsible for anxiety disorders. Owing to the limitations of the current between-subject study design and the high levels of variation observed in the behavioral and neural data among subjects, we could not examine whether the amygdala driving inputs could mediate the treatment effects on the behavioral outcome. This point should be addressed by a withinsubject study design in a future research study. In addition, the healthy control subjects in our study did not receive cortisol. Although we have previously shown that cortisol administration does not affect subjective fear response in healthy subjects (Soravia et al, 2009), we could not exclude the mere medication effect in the comparison between the cortisol group and the healthy control group.

The current study combines a pharmacological intervention with a network analysis to unravel the pharmacological targets of neural networks, although glucocorticoid receptors are ubiquitously expressed in the brain (Myers et al, 2014). Because the patients treated with cortisol did not have the driving inputs into the amygdala, we consider that glucocorticoids limited the information flow to the amygdala. The subcortical pathway, which allows the rapid processing of potential threats (Ohman et al, 2007), may be associated with the hypervigilant state that is linked to anxiety disorders (Michalowski et al, 2015) and is consistent with our results, showing that there is an association between the driving inputs into amygdala and the severity of phobiarelated symptoms. In addition, the hypervigilant state observed in patients with phobias can be associated with the hyperactivation of the amygdala (Lipka et al, 2013). Thus, glucocorticoids might be beneficial to phobia patients by reducing the hypervigilant state and by attenuating the subcortical input into the amygdala. Cortisol further modulated interactions between the fusiform gyrus and the amygdala: turning the positive fusiform-amygdala connectivity to negative. The fusiform gyrus has been implicated in spatial-associative-guided memory retrieval (de Rover et al, 2008). Thus, the cortisol-induced suppression of fusiformamygdala connectivity might be related to the well-known inhibiting effects of cortisol on memory retrieval. There are several limitations in the current study. Our female subjects participated in the study during their luteal phase. As previous studies showed that amygdala activation in response to psychological stressor depends on menstrual cycles (Chung et al, 2016) and that cortisol responses to social stress are comparable between females in a luteal phase and males (Kirschbaum et al, 1999), we investigated on female participants only in their luteal phase. However, no conclusion regarding the neural responses is drawn from the current study. Thus, our finding may not be applied to females during the follicular phase or males. In addition, we defined the ROIs of the DCM analysis based on both female and male subjects, whereas the DCM analysis has been performed only with female subject. Because the numbers of male participants were highly different among the groups, we excluded male subjects from our main DCM analysis to avoid this confound. Further studies are awaited to investigate the generalizability of the current findings. Another potential confound in the current study is that we did not strictly control for smoking among the participants. Although smoking increases the plasma levels of endogenous cortisol (Badrick et al, 2007), the administration of cortisol in the current study elevated the plasma cortisol level to a far 
greater extent than the variations caused by smoking. Therefore, we believe the cortisol administration is primarily responsible to the observed changes in the amygdala networks.

In summary, using a model-based connectivity analysis, we have revealed the neural circuits that play a crucial role in the pathophysiology of spider phobia. The driving input into amygdala is modulated by glucocorticoid administration and associated with phobia-related symptom severity. Furthermore, the framework of the current study can also be used to identify the pathophysiology and treatment targets of broader psychiatric disorders.

\section{FUNDING AND DISCLOSURE}

This work was supported by a grant from the Swiss National Science Foundation (32003B_124947), a grant from the Medical Faculty of the University of Bern (520.10), and a PRESTO grant from the Japan Science and Technology Agency. The authors declare no conflict of interest.

\section{ACKNOWLEDGMENTS}

We thank Yoan Mihov for his comments on the previous version of the manuscript, and Melanie Fisler, Basil Preisig, Joëlle Witmer, and Yvonne Renevey for their excellent research assistance.

\section{REFERENCES}

Aerni A, Traber R, Hock C, Roozendaal B, Schelling G, Papassotiropoulos A et al (2004). Low-dose cortisol for symptoms of posttraumatic stress disorder. Am J Psychiatry 161: 1488-1490.

Ahs F, Pissiota A, Michelgård A, Frans O, Furmark T, Appel L et al (2009). Disentangling the web of fear: amygdala reactivity and functional connectivity in spider and snake phobia. Psychiatry Res 172: $103-108$

American Psychiatric Association (1997). Diagnostic and statistical manual of mental disorders: DSM-IV. American Psychiatric Association: Washington.

Badrick E, Kirschbaum C, Kumari M (2007). The relationship between smoking status and cortisol secretion. J Clin Endocrinol Metab 92: 819-824.

Becker B, Mihov Y, Scheele D, Kendrick KM, Feinstein JS, Matusch A et al (2012). Fear processing and social networking in the absence of a functional amygdala. Biol Psychiatry 72: 70-77.

Buades-Rotger M, Serfling G, Harbeck B, Brabant G, Krämer UM (2016). Prednisolone increases neural reactivity to negative socioemotional stimuli in healthy young men. Eur Neuropsychopharmacol J Eur Coll Neuropsychopharmacol 26: 1176-1189.

Chan S, Debono M (2010). Replication of cortisol circadian rhythm: new advances in hydrocortisone replacement therapy. Ther $A d v$ Endocrinol Metab 1: 129-138.

Chung KC, Peisen F, Kogler L, Radke S, Turetsky B, Freiherr J et al (2016). The influence of menstrual cycle and androstadienone on female stress reactions: an fMRI study. Front Hum Neurosci 10: 44 .

de Quervain DJ, Roozendaal B, McGaugh JL (1998). Stress and glucocorticoids impair retrieval of long-term spatial memory. Nature 394: 787-790.

de Quervain DJ, Roozendaal B, Nitsch RM, McGaugh JL, Hock C (2000). Acute cortisone administration impairs retrieval of longterm declarative memory in humans. Nat Neurosci 3: 313-314. de Quervain DJ-F, Aerni A, Schelling G, Roozendaal B (2009). Glucocorticoids and the regulation of memory in health and disease. Front Neuroendocrinol 30: 358-370.

de Quervain DJ-F, Bentz D, Michael T, Bolt OC, Wiederhold BK, Margraf J et al (2011). Glucocorticoids enhance extinction-based psychotherapy. Proc Natl Acad Sci USA 108: 6621-6625.

de Quervain DJ-F, Margraf J (2008). Glucocorticoids for the treatment of post-traumatic stress disorder and phobias: a novel therapeutic approach. Eur J Pharmacol 583: 365-371.

de Rover M, Petersson KM, van der Werf SP, Cools AR, Berger HJ, Fernández G (2008). Neural correlates of strategic memory retrieval: differentiating between spatialassociative and temporal-associative strategies. Hum Brain Mapp 29: 1068-1079.

Dickerson SS, Kemeny ME (2004). Acute stressors and cortisol responses: a theoretical integration and synthesis of laboratory research. Psychol Bull 130: 355-391.

Dolan RJ, Vuilleumier P (2003). Amygdala automaticity in emotional processing. Ann NY Acad Sci 985: 348-355.

Eckstein M, Becker B, Scheele D, Scholz C, Preckel K, Schlaepfer TE et al (2014). Oxytocin Facilitates the Extinction of Conditioned Fear in Humans. Biol Psychiatry 78: 194-202.

Essau CA, Wittchen H-U, Pfister H (1999). DIA-X-Interview. Diagnostica 45: 163-164.

Etkin A, Egner T, Peraza DM, Kandel ER, Hirsch J (2006). Resolving emotional conflict: a role for the rostral anterior cingulate cortex in modulating activity in the amygdala. Neuron 51: 871-882.

Etkin A, Wager TD (2007). Functional neuroimaging of anxiety: a meta-analysis of emotional processing in PTSD, social anxiety disorder, and specific phobia. Am J Psychiatry 164: 1476-1488.

Fastenrath M, Coynel D, Spalek K, Milnik A, Gschwind L, Roozendaal B et al (2014). Dynamic modulation of amygdalahippocampal connectivity by emotional arousal. J Neurosci Off $J$ Soc Neurosci 34: 13935-13947.

Feinstein JS (2013). Lesion studies of human emotion and feeling. Curr Opin Neurobiol 23: 304-309.

Franke GH (1995). Die Symptom-Checkliste von Derogatis: Manual. SCL-90-R; deutsche Version. Beltz Test: Göttingen.

Friston KJ, Harrison L, Penny W (2003). Dynamic causal modelling. NeuroImage 19: 1273-1302.

Goense JBM, Logothetis NK (2008). Neurophysiology of the BOLD fMRI signal in awake monkeys. Curr Biol 18: 631-640.

Hariri AR, Drabant EM, Munoz KE, Kolachana BS, Mattay VS, Egan MF et al (2005). A susceptibility gene for affective disorders and the response of the human amygdala. Arch Gen Psychiatry 62: 146-152.

Hartley CA, Phelps EA (2012). Anxiety and decision-making. Biol Psychiatry 72: 113-118.

Hauner KK, Mineka S, Voss JL, Paller KA (2012). Exposure therapy triggers lasting reorganization of neural fear processing. Proc Natl Acad Sci USA 109: 9203-9208.

Hurlemann R, Rehme AK, Diessel M, Kukolja J, Maier W, Walter H et al (2008). Segregating intra-amygdalar responses to dynamic facial emotion with cytoarchitectonic maximum probability maps. J Neurosci Methods 172: 13-20.

Kirschbaum C, Kudielka BM, Gaab J, Schommer NC, Hellhammer DH (1999). Impact of gender, menstrual cycle phase, and oral contraceptives on the activity of the hypothalamus-pituitary-adrenal axis. Psychosom Med 61: 154-162.

Labuschagne I, Phan KL, Wood A, Angstadt M, Chua P, Heinrichs M et al (2010). Oxytocin attenuates amygdala reactivity to fear in generalized social anxiety disorder. Neuropsychopharmacol Off Publ Am Coll Neuropsychopharmacol 35: 2403-2413.

Lipka J, Hoffmann M, Miltner WHR, Straube T (2013). Effects of cognitive-behavioral therapy on brain responses to subliminal and supraliminal threat and their functional significance in specific phobia. Biol Psychiatry 76: 869-877. 
Luo Q, Holroyd T, Jones M, Hendler T, Blair J (2007). Neural dynamics for facial threat processing as revealed by gamma band synchronization using MEG. NeuroImage 34: 839-847.

Marle HJF, van, Hermans EJ, Qin S, Overeem S, Fernández G (2013). The effect of exogenous cortisol during sleep on the behavioral and neural correlates of emotional memory consolidation in humans. Psychoneuroendocrinology 38: 1639-1649.

Michalowski JM, Pané-Farré CA, Löw A, Hamm AO (2015). Brain dynamics of visual attention during anticipation and encoding of threat- and safe-cues in spider-phobic individuals. Soc Cogn Affect Neurosci 10: 1177-1186.

Mogg K, Bradley BP, de Bono J, Painter M (1997). Time course of attentional bias for threat information in non-clinical anxiety. Behav Res Ther 35: 297-303.

Montoya ER, Bos PA, Terburg D, Rosenberger LA, van Honk J (2014). Cortisol administration induces global down-regulation of the brain's reward circuitry. Psychoneuroendocrinology 47: 31-42.

Morris JS, Ohman A, Dolan RJ (1999). A subcortical pathway to the right amygdala mediating 'unseen' fear. Proc Natl Acad Sci USA 96: $1680-1685$.

Myers B, McKlveen JM, Herman JP (2014). Glucocorticoid actions on synapses, circuits, and behavior: implications for the energetics of stress. Front Neuroendocrinol 35: 180-196.

Ohman A, Carlsson K, Lundqvist D, Ingvar M (2007). On the unconscious subcortical origin of human fear. Physiol Behav 92: 180-185.

Pape H-C, Pare D (2010). Plastic synaptic networks of the amygdala for the acquisition, expression, and extinction of conditioned fear. Physiol Rev 90: 419-463.

Peer JM, van, Spinhoven P, Roelofs K (2010). Psychophysiological evidence for cortisol-induced reduction in early bias for implicit social threat in social phobia. Psychoneuroendocrinology 35: 21-32.

Pessoa L, Adolphs R (2010). Emotion processing and the amygdala: from a 'low road' to 'many roads' of evaluating biological significance. Nat Rev Neurosci 11: 773-783.

Phelps EA (2006). Emotion and cognition: insights from studies of the human amygdala. Annu Rev Psychol 57: 27-53.

Phelps EA, LeDoux JE (2005). Contributions of the amygdala to emotion processing: from animal models to human behavior. Neuron 48: 175-187.
Rubio-Stipec M, Bravo M, Canino G (1991). The Composite International Diagnostic Interview (CIDI): an epidemiologic instrument suitable for using in conjunction with different diagnostic systems in different cultures. Acta Psiquiátrica Psicológica América Lat 37: 191-204.

Schienle A, Schäfer A, Stark R, Vaitl D (2009). Long-term effects of cognitive behavior therapy on brain activation in spider phobia. Psychiatry Res 172: 99-102.

Soares JJ, Ohman A (1993). Preattentive processing, preparedness and phobias: effects of instruction on conditioned electrodermal responses to masked and non-masked fear-relevant stimuli. Behav Res Ther 31: 87-95.

Soravia LM, de Quervain DJ-F, Heinrichs M (2009). Glucocorticoids do not reduce subjective fear in healthy subjects exposed to social stress. Biol Psychol 81: 184-188.

Soravia LM, Heinrichs M, Aerni A, Maroni C, Schelling G, Ehlert U et al (2006). Glucocorticoids reduce phobic fear in humans. Proc Natl Acad Sci USA 103: 5585-5590.

Soravia LM, Heinrichs M, Winzeler L, Fisler M, Schmitt W, Horn H et al (2014). Glucocorticoids enhance in vivo exposure-based therapy of spider phobia. Depress Anxiety 31: 429-435.

Spielberger CD, Gorsuch RL, Lushene RE (1970). Manual for the State-Trait Anxiety Inventory. Consulting Psychologists Press: Palo Alto, CA.

Stephan KE, Penny WD, Moran RJ, Ouden HEM, den, Daunizeau J, Friston KJ (2010). Ten simple rules for dynamic causal modeling. NeuroImage 49: 3099-3109.

Straube T, Mentzel H-J, Miltner WHR (2006). Neural mechanisms of automatic and direct processing of phobogenic stimuli in specific phobia. Biol Psychiatry 59: 162-170.

Szymanski J, O’Donohue W (1995). Fear of Spiders Questionnaire. J Behav Ther Exp Psychiatry 26: 31-34.

Van Le Q, Isbell LA, Matsumoto J, Nguyen M, Hori E, Maior RS et al (2013). Pulvinar neurons reveal neurobiological evidence of past selection for rapid detection of snakes. Proc Natl Acad Sci USA 110: 19000-19005.

Veltman DJ, Tuinebreijer WE, Winkelman D, Lammertsma AA, Witter MP, Dolan RJ et al (2004). Neurophysiological correlates of habituation during exposure in spider phobia. Psychiatry Res 132: $149-158$.

Supplementary Information accompanies the paper on the Neuropsychopharmacology website (http://www.nature.com/npp) 